\title{
Populations drifting apart
}

The continued emergence of antibiotic-resistant pathogens is a major global health concern, and colistin is one of the last-line antibiotics to treat infections caused by bacteria that are resistant to most or all other antibiotics. Enterobacter cloacae is an opportunistic Gram-negative bacterium that can cause hospital-acquired infections. However, colistin-resistant strains have emerged and, in addition, antibiotic therapy sometimes fails even against susceptible bacteria. Such resistance can be due to persistence but in a new study, Band, Crispell et al. identified a small resistant bacterial subpopulation that is distinct from persisters and is responsible for the failure of antibiotic treatment in vivo.

The authors isolated a strain of E. cloacae from a renal transplant recipient and found that a small subpopulation was highly resistant to colistin. Using a deep-sequencing approach, the authors showed that bacteria grown with and without antibiotic are genotypically identical, which suggests that the resistance

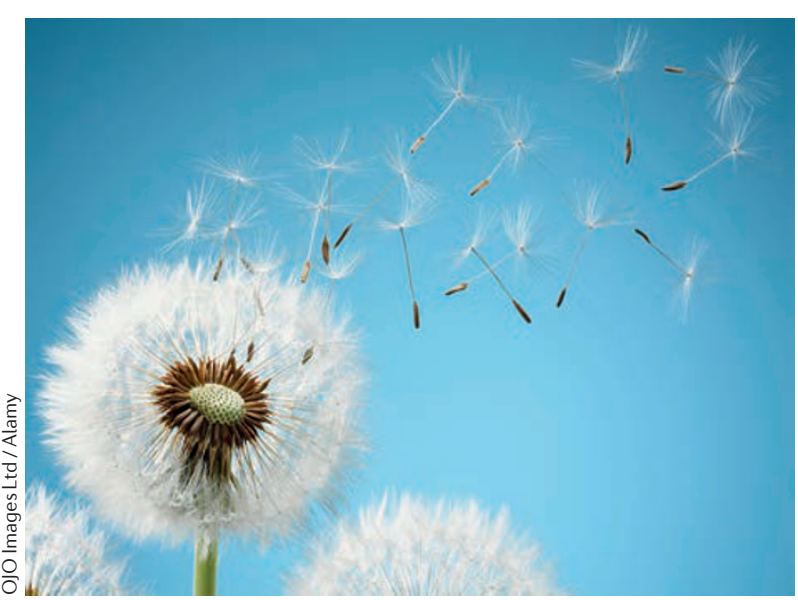

phenotype is not the result of a stable mutation. Importantly, the proportion of the resistant subpopulation increased following exposure to the antibiotic both in vitro and in vivo. This finding suggests that the resistant subpopulation is distinct from persisters, which do not increase in number in the presence of antibiotics.

Next, the authors infected wildtype mice or mice that were depleted of macrophages with the clinical isolate and showed that the resistant subpopulation increased in the wild-type mice but not in the mice that lacked macrophages. Moreover, the colistin-resistant subpopulation rapidly increased in infected macrophages, in a manner that was dependent on the internalization of the bacteria. This suggests that macrophages are both necessary and sufficient for the increase in frequency of the resistant subpopulation. The increase in the resistant subpopulation that was observed in wild-type mice was abrogated in mice that lacked lysozyme, cathelicidin-related antimicrobial peptide (CRAMP) and a functional NADPH oxidase, which indicates that these innate immune antimicrobial factors in the host have a role in the establishment of the antibioticresistant subpopulation during infection. In addition, the authors were able to show that this subpopulation is responsible for host lethality even in the presence of antibiotic, a characteristic that again distinguishes this resistance phenotype from persisters, which only grow and become pathogenic after withdrawal of the antibiotic.
RNA-sequencing analysis revealed that the resistant subpopulation had increased levels of expression of genes that are regulated by PhoQ, which is a histidine kinase that has a role in the modification of the lipid A portion of lipopolysaccharide. Indeed, the level of modified lipid A was increased in the resistant subpopulation, but this increase was not observed in a deletion mutant that lacked $p h o Q$. This, together with the findings that the $\Delta p h o Q$ strain exhibited a complete absence of the resistant subpopulation and that the mutant strain was susceptible to treatment with colistin in vivo, implies a crucial role for PhoQ in the resistance phenotype.

Finally, the authors identified another clinical isolate that harboured a colistin-resistant subpopulation and caused a lethal, antibiotic-resistant infection. However, this subpopulation was not detected during routine diagnostic testing, which highlights the need for improved diagnostic strategies to detect antibiotic-resistant subpopulations and prevent treatment failure.

In summary, this study identifies a non-genetic colistin-resistant subpopulation that is distinct from persisters as it causes acute infection and lethality during the course of treatment with antibiotics. The authors propose to refer to this resistance phenomenon as clonal heteroresistance to describe a resistant subpopulation that exhibits an increased level of antibiotic resistance relative to the larger susceptible subpopulation; with the two populations being genetically identical.

Andrea Du Toit

ORIGINAL ARTICLE Band, V. I., Crispell, E. K. et a Antibiotic failure mediated by a resistant subpopulation in Enterobacter cloacae.

Nat. Microbiol. http://dx.doi.org/10.1038/ NMICROBIOL.2016.53 (2016) 